\title{
Fibulin-3 may improve vascular health through inhibition of MMP-2/9 and oxidative stress in spontaneously hypertensive rats
}

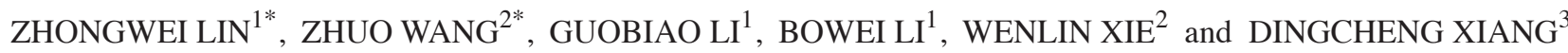 \\ ${ }^{1}$ Department of Cardiology, The First Affiliated Hospital of Guangdong Pharmaceutical University, Guangzhou, \\ Guangdong 510000; ${ }^{2}$ Department of Pathology, The First Affiliated Hospital of Sun Yat-sen University, Guangzhou, \\ Guangdong 510080; ${ }^{3}$ Department of Cardiology, Guangzhou General Hospital of Guangzhou Military Command, \\ Southern Medical University, Guangzhou, Guangdong 510110, P.R. China
}

Received April 21, 2015; Accepted March 1, 2016

DOI: $10.3892 / \mathrm{mmr} .2016 .5036$

\begin{abstract}
Fibulin-3 has been suggested to function in the remodeling of the extracellular matrix, however its role remains unclear in hypertensive vascular remodeling. In the current study, 10 Wistar-Kyoto (WKY) rats (control group) and 30 spontaneously hypertensive rats (SHRs) were used. SHRs were randomized into three groups: The placebo group, intravenous (I.V.) physiological saline; the FBLN-1 group, low-dose fibulin-3 protein (I.V.; $120 \mathrm{ng} / \mathrm{kg}$ ); and the FBLN-2 group, high-dose fibulin-3 protein (I.V.; $240 \mathrm{ng} / \mathrm{kg}$ ). Histological analysis was used to analyze vascular remodeling. The expression of fibulin-3, matrix metalloproteinase (MMP)-2, MMP-9 and tissue inhibitor of metalloproteinase (TIMP)-3 were detected by immunohistochemistry, western blotting and reverse transcription-quantitative polymerase chain reaction. Oxidative stress was detected by dihydroethidium staining. The systolic blood pressure (SBP) of SHRs was observed to be significantly greater than that of WKY rats $(\mathrm{P}<0.05)$. SBP in the FBLN-2 group was significantly reduced compared with the placebo group $(182 \pm 12 \mathrm{mmHg}$ vs. $224 \pm 14 \mathrm{mmHg}$; $\mathrm{P}<0.05)$. The thoracic aortic wall thickness in the SHR groups (placebo group, FBLN-1 group and FBLN-2 group) was observed to the significantly thicker than in the control group $(\mathrm{P}<0.01)$. The wall thickness of the FBLN-2 group was significantly greater than that of the placebo and FBLN-1 groups $(124.2 \pm 11.8 \mu \mathrm{m}$ vs. $106.9 \pm 9.5 \mu \mathrm{m}$ and $96.8 \pm 10.2 \mu \mathrm{m} ; \mathrm{P}<0.05)$. The wall-to-lumen ratios of the placebo, FBLN-1 and FBLN-2 groups were significantly greater than that of the control
\end{abstract}

Correspondence to: Dr Zhongwei Lin, Department of Cardiology, The First Affiliated Hospital of Guangdong Pharmaceutical University, 19 Nonglinxia Road, Guangzhou, Guangdong 510000, P.R. China

E-mail: gdpcxn3110@163.com

*Contributed equally

Key words: fibulin-3, matrix metalloproteinase, oxidative stress, hypertension, vascular remodeling group $(\mathrm{P}<0.05)$. In addition, the expression levels of fibulin-3 and MMP-2/9 at protein and mRNA levels were significantly increased in the thoracic aorta of the placebo group compared with the control group $(\mathrm{P}<0.05)$. The levels of MMP-2/9 were significantly reduced in the FBLN-2 group compared with the placebo group $(\mathrm{P}<0.05)$. Levels of TIMP-3 however, exhibited no significant differences in the four groups $(\mathrm{P}>0.05)$. Reactive oxygen species (ROS) were increased in the placebo group vs. the control group. Fibulin-3 was able to alleviate the levels of ROS in the FBLN groups. It is suggested that fibulin-3 may act as a growth factor in the arteries. In addition, the results indicated that fibulin-3 may reduce the levels of MMP-2 and -9 and oxidative stress in hypertensive vascular remodeling. Upregulating fibulin-3 may be beneficial for improving vascular health and offsetting certain cardiovascular risk factors of hypertension.

\section{Introduction}

The fibulins are a family of seven extracellular matrix (ECM) proteins characterized by tandem arrays of epidermal growth factor-like domains and a C-terminal fibulin-type module (1). They are widely distributed and often associated with vasculature and elastic tissues (2). Fibulin-3 has been reported to be widely distributed in the ECM in vertebrates and is essential for normal development and maintenance of the microenvironment of embryonic and adult tissues.

Matrix metalloproteinases (MMPs) have been reported to serve significant roles in the degradation of ECM components (3). Tissue inhibitor of metalloproteinase (TIMP), which binds to MMP with a 1:1 molar stoichiometry, is an endogenous inhibitor of MMPs and regulates matrix remodeling by MMPs. Fibulin-3 is able to target endothelial cellular expression of MMPs and TIMPs, thereby has been suggested to reduce ECM proteolysis and remodeling (4). Remodeling of ECM is an important regulator of physiological and pathological processes in the vessel wall (5). Tumors and cultured cells overexpressing fibulin-3 have been demonstrated to exhibit elevated expression levels and activity of MMPs, such as MMP-2 and MMP-9 (6). A previous study reported that fibulin-3 negatively modulated the invasiveness of lung cancer cells via regulation of MMP-7 and -2 (7). An additional study observed that fibulin-3 was able 
to reduce MB114 endothelial cell expression of MMP-2 and -3 while simultaneously increasing the expression of the MMP inhibitors TIMP-1 and -3 (8).

However, the role of fibulin-3 in hypertensive vascular remodeling remains unclear. The current study aimed to elucidate the association between fibulin-3, MMPs and hypertensive vascular remodeling in a hypertensive animal model. In the current study, the presence of fibulin-3, MMP-2, MMP-9 and TIMP-3 in the aortas of spontaneously hypertensive rats (SHR) was analyzed. Furthermore, the influence of injected fibulin-3 protein was measured. In addition, the association between fibulin-3 and oxidative stress in vascular remodeling in hypertension was investigated.

\section{Materials and methods}

Animals and blood pressure measurement. The experimental protocols were approved by the Guangdong Pharmaceutical University Animal Care and Use Committee and were performed in accordance with the Sun Yat-sen University Guidelines for the Care and Use of Laboratory Animals. Wistar-Kyoto (WKY) rats (8-week-old; $n=10)$ and spontaneously hypertensive rats (SHRs) (8-week-old; n=30) weighing 140-160 g were used. The rats were kept under a 12/12 h light cycle, with specific pathogen free conditions and $5 \mathrm{~g}$ food and 6-7 $\mathrm{ml}$ water/100 g body weight. All rats were purchased from Beijing Vital River Experimental Animal Technology Co., Ltd. (Beijing, China). The rats were divided into four groups $(n=10 /$ group): Control group, WKY rats with no treatment; placebo group, SHRs treated with intravenous (I.V.) physiological saline; FBLN-1 group, SHRs treated with low-dose fibulin-3 protein (I.V.; $120 \mathrm{ng} / \mathrm{kg}$ ); and the FBLN-2 group, SHRs treated with high-dose fibulin-3 protein (I.V.; $240 \mathrm{ng} / \mathrm{kg}$ ).

Rats were injected with physiological saline or fibulin-3 protein through the tail vein once per week for 8 weeks. Blood pressure was serially determined in conscious, trained rats using a noninvasive tail-cuff device (Anhui Zhenghua Biological instrument Equipment Co., Ltd., Anhui, China) by a researcher blinded to the groups. All rats were sacrificed with an overdose of pentobarbital subsequent to 8 weeks of treatment. The tissue samples from the aorta were then collected.

Histological analysis. The thoracic aortas were removed and fixed in $4 \%$ paraformaldehyde (pH 7.4; Guangzhou Zhanchen Biological Technology Co., Ltd., Guangzhou, China) or liquid nitrogen for protein isolation and western blotting. Fixed tissues were sectioned and stained with hematoxylin and eosin (Sigma-Aldrich, St. Louis, MO, USA) for the evaluation of pathological alterations. The wall-to-lumen (W/L) ratio was evaluated by the ratio of wall thickness to the internal diameter of the lumen. The wall thicknesses and internal diameter of lumen were assessed using Image-Pro Plus software, version 6.0 (Media Cybernetics, Inc., Rockville, MD, USA).

Immunohistochemistry. Immunohistochemistry of sections from paraffin-embedded tissue blocks (Thermo Fisher Scientific, Inc., Waltham, MA, USA) was conducted according to standard protocols in $5 \mu \mathrm{m}$-sections of thoracic aorta. Briefly, sections were deparaffinized in xylene (Guangzhou Zhanchen Biological Technology Co., Ltd.) and rehydrated using a series of graded alcohols (Guangzhou Zhanchen Biological Technology Co., Ltd.). The sections were then treated with 3\% hydrogen peroxide (Guangzhou Zhanchen Biological Technology Co., Ltd.) for $10 \mathrm{~min}$ to quench endogenous peroxidase activity. The antigens were retrieved in $0.01 \mathrm{M}$ sodium citrate buffer (pH 6.0; Guangzhou Zhanchen Biological Technology Co., Ltd.) using a microwave oven. The sections were incubated overnight with the appropriate primary antibody in a humidified container at $4^{\circ} \mathrm{C}$, subsequent to $30 \mathrm{~min}$ preincubation in $10 \%$ normal goat serum (Sigma-Aldrich) to prevent nonspecific staining. The negative control was phosphate-buffered saline (PBS; Guangzhou Zhanchen Biological Technology Co., Ltd.), used instead of the primary antibody. The sections were incubated with the rabbit polyclonal EnVision-horseradish peroxidase (HRP)-conjugated secondary antibody (K5007; Dako, Glostrup, Denmark) for $30 \mathrm{~min}$ at room temperature. Tthe tissue slides were then treated with a nonbiotin HRP detection system according to the manufacturer's instructions. The slides were then counterstained with hematoxylin. The results were considered positive when a brown precipitate was observed to have developed in the cytoplasm. The following primary antibodies were used: Polyclonal rabbit anti-fibulin-3 (AP9095a; 1:50), monoclonal mouse anti-MMP-2 (A-AJ1497b; 1:300), monoclonal mouse anti-MMP-9 (A-AJ1503b; 1:50) and rabbit polyclonal TIMP-3 (A-AO1052a; 1:50) (all from Abgent, Inc., San Diego, CA, USA). A total of five positive expression fields from each section were selected at random in triplicate, and then the positive regions were analyzed in Image-Pro Plus software, version 6.0, to determine the integral optical density and area. The average optical density was calculated, and the average of five optical density values was determined to represent the expression intensity in the section.

Protein isolation and western blotting. Aortas were dissected and harvested ( 3 pooled aortas per experiment). Total proteins were isolated from the aorta using a protein extraction reagent (Nanjing KeyGen Biotech. Co., Ltd., Nanjing, China). Samples were lysed on ice for $1 \mathrm{~h}$ with lysis buffer [1X PBS, 1\% NP40, $0.1 \%$ sodium dodecyl sulfate, $5 \mathrm{mM}$ ethylenediaminetetraacetic acid, $0.5 \%$ sodium deoxycholate and $1 \mathrm{mM}$ sodium orthoyanadate] containing $1 \%$ protease inhibitor phenylmethanesulfonyl fluoride (Nanjing KeyGen Biotech. Co., Ltd.), and clarified by centrifugation at $14,000 \times \mathrm{g}$ for $10 \mathrm{~min}$ at $4^{\circ} \mathrm{C}$. The protein contents of the cleared lysates were determined using a Bicinchoninic Acid Protein Quantitative Analysis kit (Beijing CoWin Biotech Co., Ltd., Beijing, China). The protein bands were transferred to polyvinylidene difluoride membranes (EMD Millipore, Billerica, MA, USA) which were pretreated with methanol. The membranes were then incubated with primary antibodies overnight at $4^{\circ} \mathrm{C}$ and then with the appropriate secondary antibody, as follows: Rabbit polyclonal EnVision-HRP-conjugated secondary antibody (K5007), rabbit IgG polyclonal-HRP (ASS1003; 1:5,000; Abgent, Inc.) or rabbit IgM polyclonal-HRP (ASS1005; 1:5,000; Abgent, Inc.). The primary antibodies mentioned above against fibulin-3 (1:250), MMP-2 (1:1,000), MMP-9 $(1: 1,000)$ and TIMP-3 $(1: 1,000)$ were used.

Total RNA extraction and reverse transcriptase-quantitative polymerase chain reaction (RT-qPCR). A $3 \mathrm{~mm}$-long section 
of fresh aorta was obtained at the time of surgical resection and was immediately frozen at $-80^{\circ} \mathrm{C}$ until required. The section was then prepared for RNA extraction. Total RNA was extracted using TRIzol reagent (Invitrogen; Thermo Fisher Scientific, Inc.) according to the manufacturer's instructions. For RT-qPCR, SYBR Green Supermix (Roche Diagnostics, Indianapolis, IN, USA) was used, and standard curves for each primer set were generated to confirm that only one amplicon was generated at the same efficiency as the reference gene glyceraldehyde 3-phosphate dehydrogenase (GAPDH). The sequences for the primers used for amplifying rat fibulin-3, MMP-9, MMP-2 and the internal reference GADPH were as described previously (9). RT-qPCR was performed using the following thermal cycling conditions: $10 \mathrm{~min}$ at $95^{\circ} \mathrm{C} ; 45$ cycles of denaturation at $95^{\circ} \mathrm{C}$ for $15 \mathrm{sec}$, annealing for $25 \mathrm{sec}$ at $62^{\circ} \mathrm{C}$ and elongation at $60^{\circ} \mathrm{C}$ for $1 \mathrm{~min}$; final extension for $10 \mathrm{~min}$ at $72^{\circ} \mathrm{C}$, termination of the reaction at $4^{\circ} \mathrm{C}$. Target gene expression was calculated using the $\Delta \Delta \mathrm{Cq}$ and comparative methods (10) subsequent to normalization to GAPDH expression.

Detection of oxidative stress. Reactive oxygen species (ROS) production in the thoracic aortas was detected by dihydroethidium (DHE) staining (Vigorous Biotechnology Beijing Co., Ltd., Beijing, China). Frozen, enzymatically intact, $6-\mu \mathrm{m}$-thick sections of thoracic aortas were incubated with $10 \mu \mathrm{mol} / 1 \mathrm{DHE}$ in PBS for $30 \mathrm{~min}$ at $37^{\circ} \mathrm{C}$ in a dark, humidified chamber. DHE is oxidized by ROS and converted to ethidium, which binds to DNA in the nucleus and fluoresces red. Thus, ROS production was examined under a fluorescent microscope (BX53; Olympus Corporation, Tokyo, Japan).

Statistical analysis. Data are presented as the mean \pm standard deviation. Differences between groups were analyzed using one-way analysis of variance, while differences within two groups were assessed using unpaired Student's t-tests. Statistical analysis was conducted using SPSS for Windows, version 17.0 (SPSS, Inc., Chicago, IL, USA). P<0.05 was considered to indicate a statistically significant difference.

\section{Results}

General observations. Systolic blood pressure data are presented in Table I. No significant differences in body weight and heart rates were observed between the four groups. Systolic blood pressures measured in the placebo, FBLN-1 and FBLN-2 groups were observed to be significantly greater than those in the control group. Blood pressure in the FBLN-2 group was significantly reduced compared with the placebo group $(\mathrm{P}<0.05)$.

Histological analysis of aortic structure. To investigate the effect of hypertensive vascular remodeling, vascular wall thickness and W/L ratios were investigated in the thoracic aortas of 16-week-old SHRs and 8-week-old WKY rats. Thoracic aortas were evaluated in sections stained with hematoxylin and eosin (Fig. 1). The aortas of the SHRs exhibited wall thickening and hypertrophic vascular smooth muscle cells (SMCs). The thickness of the vascular wall of all SHRs was observed to be significantly greater than that in WKY rats $(\mathrm{P}<0.05)$. FBLN-1 and FBLN-2 groups were demonstrated to exhibit significant increases in aortic wall thickness when compared with the placebo and control groups $(\mathrm{P}<0.05)$, while FBLN-2 group wall thickness was greater than that of the FBLN-1 group (Table I). The W/L ratios of the placebo, FBLN-1 and FBLN-2 groups were significantly greater than that of the control group $(\mathrm{P}<0.05)$, while no significant differences were observed between the FBLN-1, FBLN-2 and placebo groups.

Protein expression of fibulin-3, MMP-2, MMP-9 and TIMP-3 in rat aortic tissue. The alterations in protein expression levels were observed by immunohistochemistry (Fig. 2A). Quantitation of the immunohistochemical expression of fibulin-3, MMP-2, MMP-9 and TIMP-3 were assessed through average optical density using Image-Pro Plus software, version 6.0, and the data were presented as the mean \pm standard deviation (Fig. 2B). In the placebo group, fibulin-3, MMP-2 and MMP-9 were significantly increased in the thoracic aortas compared with the control group $(\mathrm{P}<0.05)$. The levels of fibulin-3 in the FBLN-2 group were greater than that of the placebo group $(\mathrm{P}<0.05)$. The levels of MMP-2 and MMP-9 were observed to be significantly reduced in the FBLN-2 group compared with the placebo group $(\mathrm{P}<0.05)$. The levels of TIMP-3 however, were not observed to be significantly different in any of the four groups.

Western blot analysis was also conducted to measure the protein expression levels of fibulin-3, MMP-2, MMP-9 and TIMP-3. The expression levels of fibulin-3 were significantly increased in the placebo, FBLN-1 and FBLN-2 groups, compared with the control group (Fig. 3A). In line with the immunohistochemistry results, the expression of MMP-2 and MMP-9 were observd to be increased in the placebo group compared with the control group, and reduced in the FBLN-treated groups compared with the placebo group (Fig. 3A). The expression levels of TIMP-3 were not significantly different in any of the four groups, as was observed in the immunohistochemistry analysis.

mRNA expression of fibulin-3, MMP-2 and MMP-9 in rat aortic tissue. In the thoracic aortas, the mRNA levels of fibulin-3, MMP-2 and MMP-9 were observed to be significantly increased in the placebo group compared with the control group. The level of fibulin-3 in the FBLN-2 group was significantly greater than that of the placebo group $(\mathrm{P}<0.05)$. The levels of MMP-2 and MMP-9 were observed to be significantly reduced in the FBLN-2 group compared with the placebo group $(\mathrm{P}<0.05$; Fig. $3 \mathrm{~B})$.

Oxidative stress in the thoracic aorta. Dihydroethidium staining indicated clear ROS generation in the thoracic aortas of the placebo, FBLN-1 and FBLN-2 groups when compared with the control group (Fig. 4). In addition, fibulin-3 protein was observed to be able to alleviate ROS in the FBLN-1 and FBLN-2 groups compared with the placebo group (Fig. 4). Little or no oxidative stress was detected in the aortas of the FBLN-1 and FBLN-2 groups (Fig. 4).

\section{Discussion}

Fibulins are ECM proteins that serve important roles in the biology of tissue organogenesis and vasculogenesis, in 
Table I. General data of the four groups.

\begin{tabular}{|c|c|c|c|c|c|c|}
\hline Group & $\mathrm{n}$ & $\begin{array}{c}\text { Body } \\
\text { weight (g) }\end{array}$ & $\begin{array}{c}\text { SBP } \\
(\mathrm{mmHg})\end{array}$ & $\begin{array}{c}\text { HR } \\
\text { (beats/min) }\end{array}$ & $\begin{array}{l}\text { Wall thickness } \\
\qquad(\mu \mathrm{m})\end{array}$ & $\begin{array}{c}\mathrm{W} / \mathrm{L} \text { ratio } \\
(\%)\end{array}$ \\
\hline Control & 10 & $264.50 \pm 15.78$ & $122 \pm 8$ & $289 \pm 11$ & $81.4 \pm 10.8$ & $6.34 \pm 0.8$ \\
\hline Placebo & 10 & $262.68 \pm 13.25$ & $224 \pm 14^{\mathrm{a}}$ & $311 \pm 11$ & $96.8 \pm 10.2^{b}$ & $7.85 \pm 0.6^{\mathrm{a}}$ \\
\hline FBLN-1 & 10 & $260.46 \pm 15.08$ & $208 \pm 11^{\mathrm{a}}$ & $310 \pm 8$ & $106.9 \pm 9.5^{\mathrm{b}, \mathrm{c}}$ & $8.03 \pm 0.7^{\mathrm{a}}$ \\
\hline FBLN-2 & 10 & $262.78 \pm 15.28$ & $182 \pm 12^{\mathrm{a}, \mathrm{b}, \mathrm{c}}$ & $302 \pm 10$ & $124.2 \pm 11.8^{\mathrm{b}, \mathrm{c}, \mathrm{d}}$ & $7.99 \pm 0.8^{a}$ \\
\hline F-value & & 1.774 & 13.014 & 1.523 & 8.658 & 18.06 \\
\hline P-value & & 0.175 & 0.000 & 0.236 & 0.000 & 0.000 \\
\hline
\end{tabular}

${ }^{\mathrm{a}} \mathrm{P}<0.05$ and ${ }^{\mathrm{b}} \mathrm{P}<0.01$ vs. the control group; ${ }^{\mathrm{C}} \mathrm{P}<0.05$ vs. the placebo group; ${ }^{\mathrm{d}} \mathrm{P}<0.05$ vs. the FBLN-1 group. SBP, systolic blood pressure; HR, heart rate; W/L ratio, wall to lumen ratio; FBLN-1, low-dose fibulin 3 group; FBLN-2, high-dose fibulin 3 group.

$\mathbf{A}$
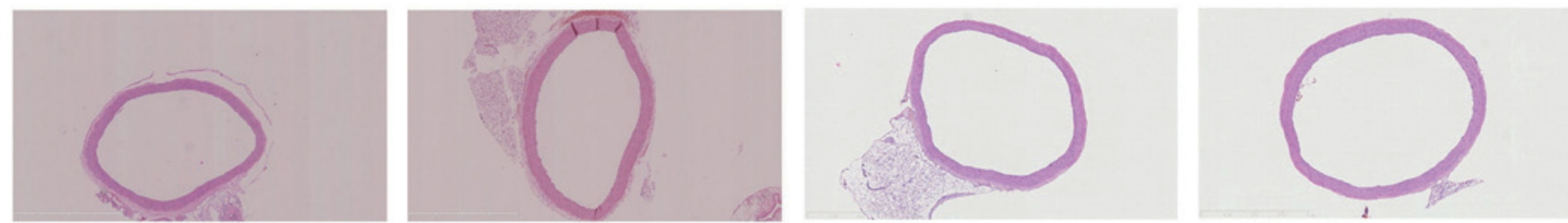

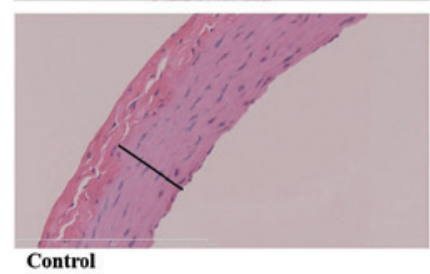

B

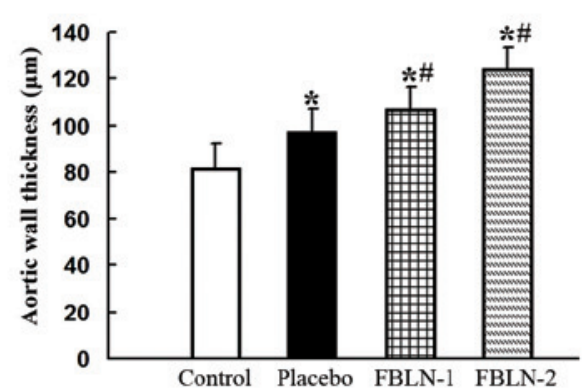

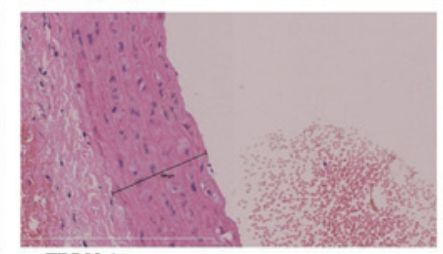

FBLN-1

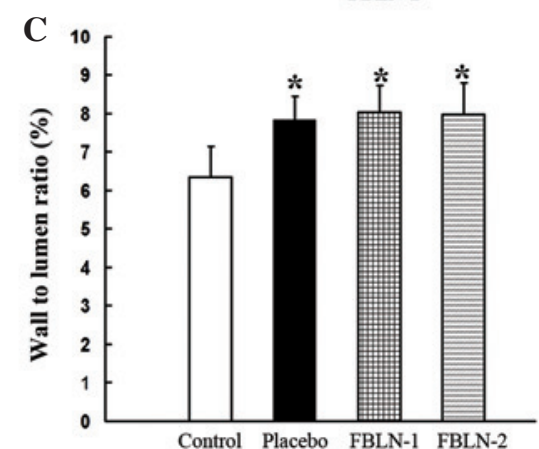

Figure 1. Histological analysis of aortic structure. (A) The thoracic aorta were stained with hematoxylin and eosin. Upper panel, magnification of x50; lower panel, magnification of x400. (B) Quantitative analysis of vascular wall thickness of the control, placebo, FBLN-1 and FBLN-2 groups. (C) Quantitative analysis for the wall-to-lumen ratios of the control, placebo, FBLN-1 and FBLN-2 groups. ${ }^{*} \mathrm{P}<0.05$, vs. control group; ${ }^{\#} \mathrm{P}<0.05$, vs. placebo group. FBLN-1, low-dose fibulin 3 group; FBLN-2, high-dose fibulin 3 group.

addition to the pathology of fibrogenesis, tumorigenesis and additional genetic diseases. Previous studies have indicated that fibulin-type modules act to mediate specific ECM binding interactions $(2,11)$. Of the seven fibulins, aortic disease-associated functions have reported for fibulin-1, -2, -4 and -5 . Fibulin-1 is deposited in human coronary artery atherosclerotic lesions in association with fibrinogen (12). The capability of fibulin-1 to inhibit cell motility may influence vascular SMC migration during vascular remodeling. Fibulin-2 has been observed to be expressed at a relatively low level in the medial layer of the blood vessels such as in the aorta (13). By contrast, the expression of fibulin-2 has been observed to be high in SMC-rich regions of atherosclerotic aortic lesions (13). Fibulin-2 had additionally been detected in mechanically injured mouse carotid arteries. These observations were consistent with a previous study which identified a fibulin-4 deficiency in mice with enlarged and tortuous aortas with intramural bleeding, aneurysm formation, aortic stiffening and aortic dissection (14). Conditional ablation of fibulin-4 expression in vascular SMCs indicated that fibulin-4 was critical for SMC differentiation, as demonstrated by the reduction of smooth muscle myosin heavy chain and $\alpha$ smooth muscle actin expression (15). Fibulin-5-deficient mice in addition to humans with a fibulin-5 deficiency have been identified to not develop aneurysms, however it has been suggested that fibulin-5 serves a role in the vascular injury response. 
A

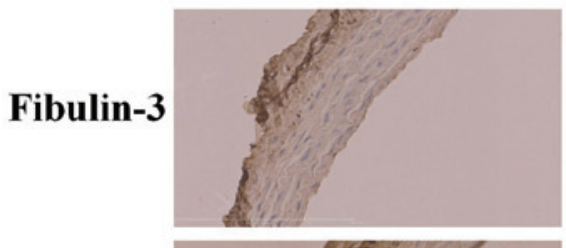

MMP-2

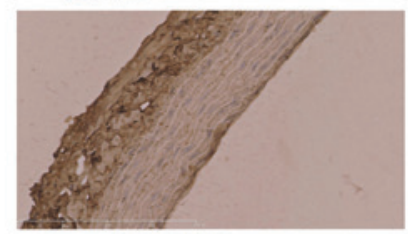

MMP-9

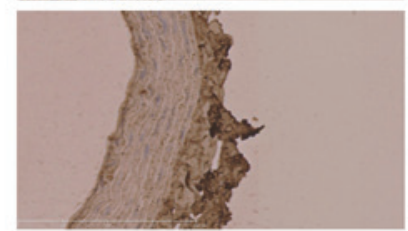

TIMP-3

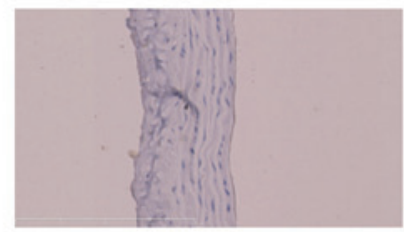

B

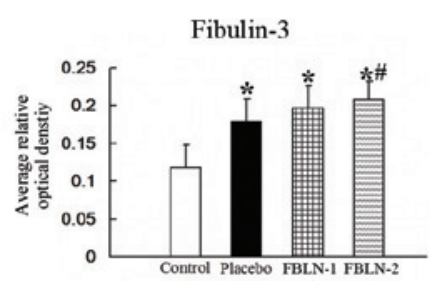

Placebo
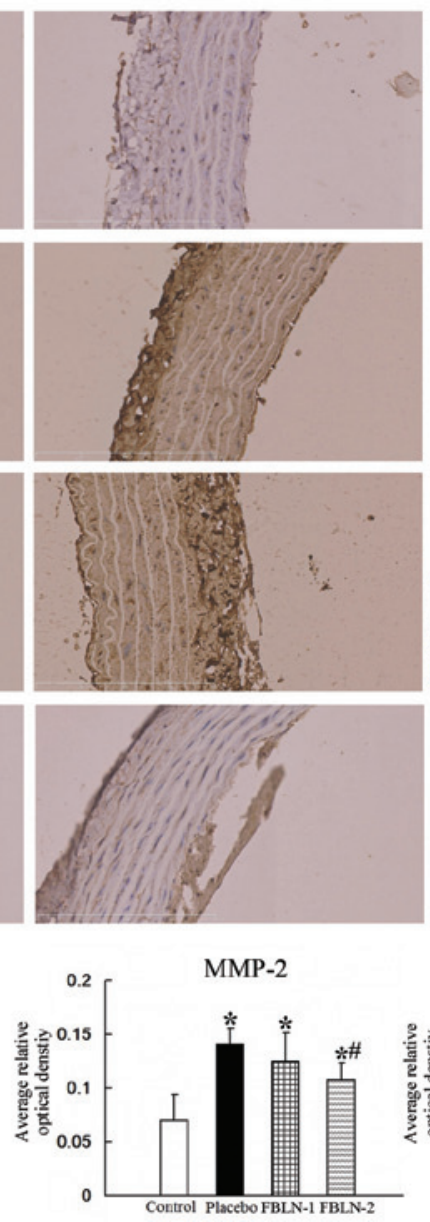

FBLN-1
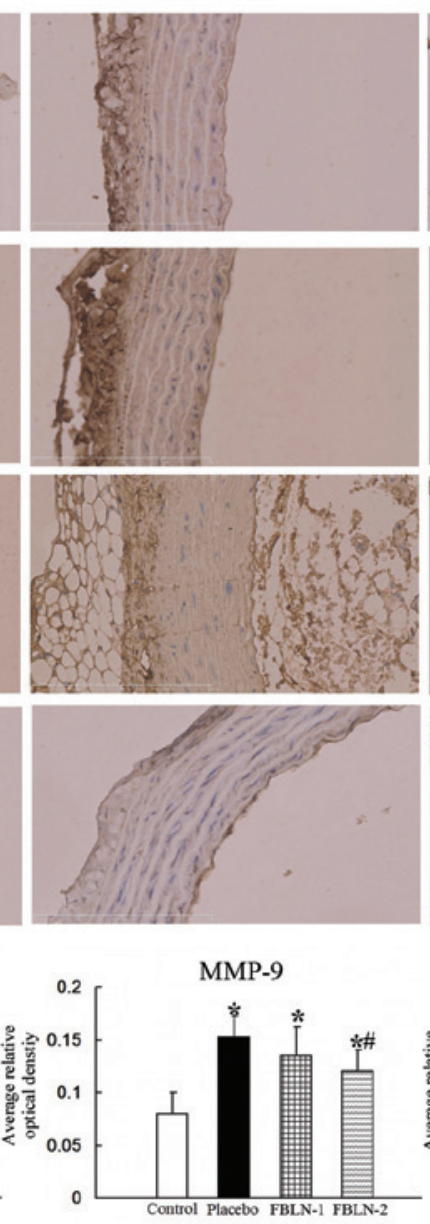

FBLN-2
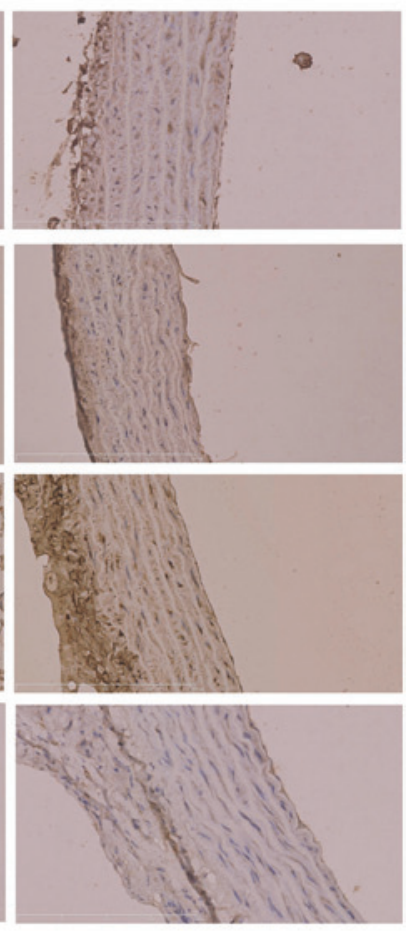

TIMP-3

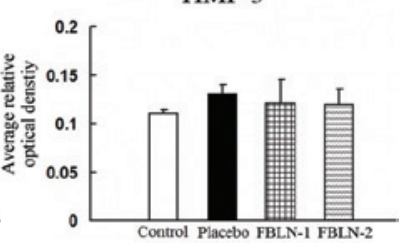

Figure 2. The expression of fibulin-3, MMP-2, MMP-9 and TIMP-3 by immunohistochemical analysis. (A) Evaluation of fibulin-3, MMP-2, MMP-9 and TIMP-3 by immunofluorescence microscopy in rat aortic control, placebo, FBLN-1 and FBLN-2 group tissues (magnification, $x 400$ ). (B) Quantitative analysis of the immunohistochemical expression of fibulin-3, MMP-2, MMP-9 and TIMP-3 assessed by the average optical density. " $\mathrm{P}<0.05$ vs. control group; ${ }^{\sharp} \mathrm{P}<0.05$ vs. placebo group. MMP, matrix metalloproteinase; TIMP-3, tissue inhibitor of metalloproteinase 3; FBLN-1, low-dose fibulin 3 group; FBLN-2, high-dose fibulin 3 group.

Fibulin-5 was observed to be upregulated 28 days following vascular injury (16). In addition, previous analysis of vascular SMCs from fibulin-5-deficient mice suggested that fibulin-5 commonly acts as a negative regulator of proliferation and migration (17).

Fibulin-3, fibulin-4 and fibulin-5 are small, monomeric proteins expressed in the ECM of blood vessels and elastic tissues (2). Fibulin-3 (also termed EFEMP1) was first isolated from the screening of differentially expressed genes between senescent and quiescent human fibroblasts. The structure of fibulin-3 is known to be similar to that of fibulin-4, however, the function of fibulin-3 in vascular SMCs remains unclear. In the current study, it was identified that upregulation of the expression of fibulin-3 and MMPs was associated with hypertensive vascular remodeling in the thoracic aortas of SHRs compared with those of WKY rats. Fibulin-3-treated arteries exhibited thicker aortic walls than those of the placebo group, while the W/L ratio of the aorta was not significantly different between the FBLN groups and the placebo group. These results indicated that the increasing level of fibulin-3 may promote growth of the vascular wall, and did not aggravate remodeling of the aorta. Therefore, the results of the current study indicate that fibulin-3 may act as a growth factor in the arteries, which is similar to the results of a previous study (17), however the mechanism remains unclear.

Vascular remodeling may be caused by SMC hypertrophy or hyperplasia, deposition of ECM elements, or a combination of these two factors (18). The current study demonstrated the hyperplasia and hypertrophy of SMCs in arteries, in addition to observing that fibulin-3, MMP-2 and MMP-9 were significantly expressed in the SMCs of the aortic wall in SHRs compared with WKY rats. These observations indicated that fibulin-3, MMP-2 and MMP-9 serve important roles in hypertensive vascular remodeling. The function of MMP-2 and MMP-9 in vascular remodeling was identified to be similar to that observed in previous studies, which indicated that increased MMP-9 and MMP-2 activity was associated with degradation of the elastic laminae of hypertensive arteries $(19,20)$. However, the role of fibulin-3 in vascular remodeling had not, to the best of our knowledge, been reported previously, thus was investigated to identify novel results.

Previous studies have demonstrated that the overexpression of fibulin-3 in tumors may increase the expression and activity of MMPs, such as MMP-2 and MMP-9, and a disintegrin and 
A

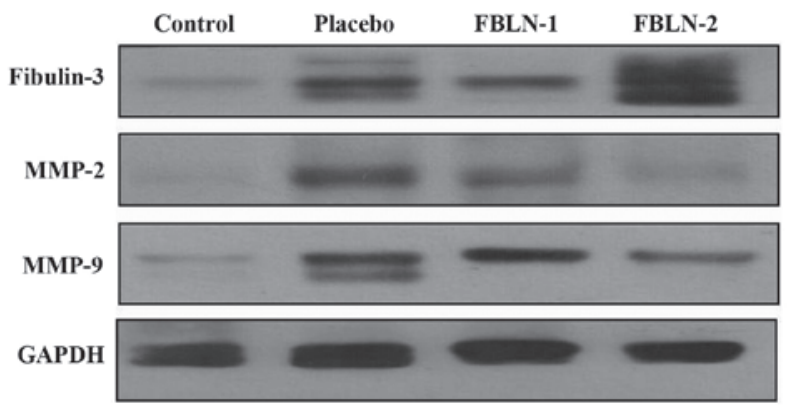

B
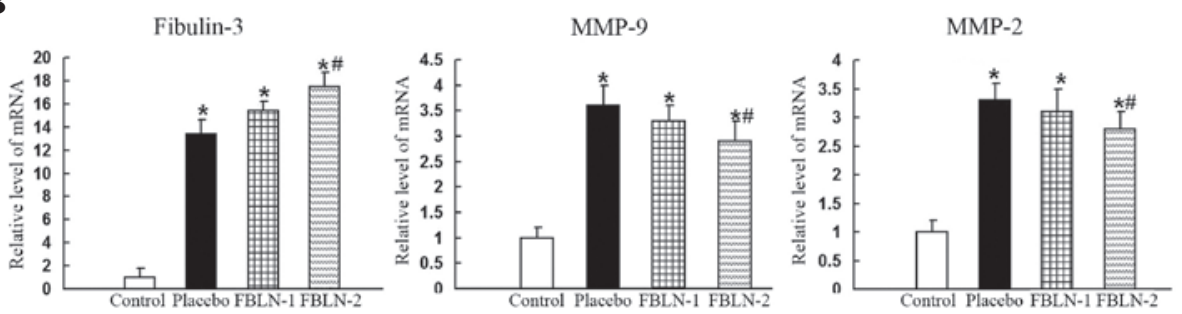

Figure 3. (A) The expression levels of fibulin-3, MMP-2 and MMP-9 by western blotting in rat aortic control, placebo, FBLN-1 and FBLN-2 group tissues. The expression levels of fibulin-3 were sequentially increased in the placebo, FBLN-1 and FBLN-2 groups, compared with the control group. Expression of MMP-2 and MMP-9 was increased in the placebo group compared with the control group. Expression of MMP-2 and MMP-9 was reduced in the FBLN-treated groups compared with the placebo group. Protein expression levels were normalized to GADPH. (B) Analysis of the mRNA expression levels of fibulin-3, MMP-9 and MMP-2 in rat aortic tissue. In the thoracic aortas, the mRNA levels of fibulin-3, MMP-2 and MMP-9 were observed to be significantly increased in the placebo group compared with the control group. The level of fibulin-3 in the FBLN-2 group was significantly greater than that of the placebo group, whereas levels of MMP-2 and -9 were significantly reduced in the FBLN-2 group compared with the placebo group. The levels of mRNA expression were normalized to GAPDH. "P<0.05 vs. control group; " $\mathrm{P}<0.05$ vs. placebo group. MMP, matrix metalloproteinase; FBLN-1, low-dose fibulin 3 group; FBLN-2, high-dose fibulin 3 group; GAPDH, glyceraldehyde 3-phosphate dehydrogenase.

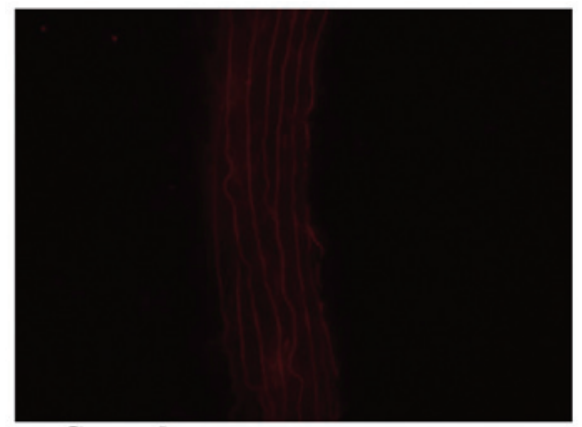

Control

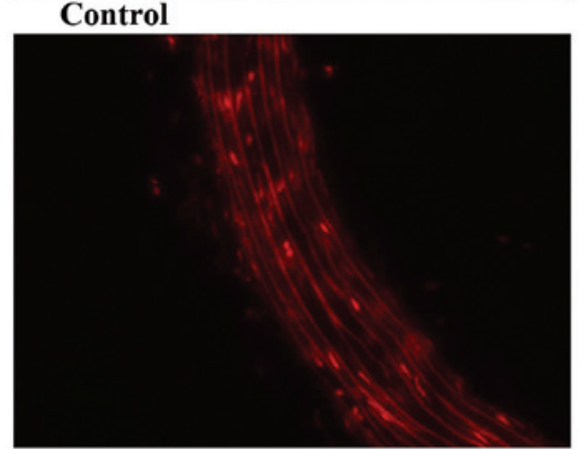

FBLN-1
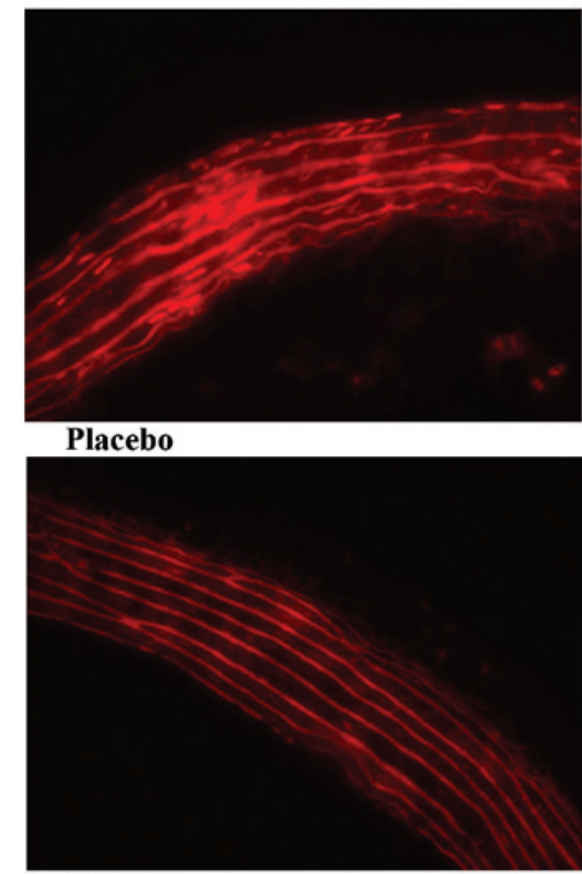

FBLN-2

Figure 4. Analysis of oxidative stress in rat thoracic aorta tissue. Dihydroethidium staining indicated clear ROS generation in thoracic aorta tissues in the placebo, FBLN-1 and FBLN-2 groups compared with the control group. ROS levels were alleviated in the FBLN-1 and FBLN-2 groups compared with the placebo group (magnification, x200). ROS, reactive oxygen species; FBLN-1, low-dose fibulin 3 group; FBLN-2, high-dose fibulin 3 group.

metalloproteinase with thrombospondin motifs $5(6,7)$. In the current study, it was identified that fibulin-3-treated aortas exhibited reduced levels of MMP-2 and MMP-9. Fibulin-3 negatively modulated the levels of MMP-2 and MMP-9 in SHRs in vivo. The result was similar to the previously identified role of fibulin-3 in non-small cell lung cancer (7). Thus, it was suggested that fibulin-3 was a negative regulator of MMP-2 and MMP-9 in arteries associated with hypertension.

The mechanisms by which fibulin-3 reduced the levels of MMPs in hypertension remain to be fully elucidated. A 
possible explanation was that activated fibulin-3 in hypertension induces the expression of TIMP-3, which is an endogenous inhibitor of MMPs and the binding partner of fibulin-3 (21). However, in the present study, no alterations in TIMP-3 in the aortic walls of WKY rats and SHRs were observed. Therefore, it was suggested that the role of fibulin-3 in MMP expression regulation was not via the TIMP-3 pathway in hypertension. Thus, further study was required to elucidate the mechanisms involved in fibulin-3-mediated regulation of MMPs.

Oxidative stress contributes to the pathology of cardiovascular disorders including hypertension (22). ROS may directly alter vascular function or cause alterations in vascular tone via several mechanisms, including altered nitric oxide bioavailability and signaling (23). Previous studies have investigated the roles of ROS and cardiovascular remodeling, which are accompanied by cellular alignment, myocyte elongation and reconstruction (24). The inductions of MMP-2, MMP-9 and oxidative stress have been previously demonstrated to be important in the development of cardiovascular disease and hypertension (25). Oxidative stress may contribute to the abnormal structure and function of elastic fibers in pathological conditions, by reducing cross-linking and interactions with other proteins required for elastic fiber assembly, including fibulin-4, fibulin-5, and fibrillin-2 (26). In the current study, the presence of oxidative stress was identified in the pathogenesis of hypertension. Furthermore, it was demonstrated that fibulin-3 was able to reduce MMP-2, MMP-9 and oxidative stress in the FBLN groups compared with the placebo group. Thus, it was suggested that increasing fibulin-3 may be beneficial for the improvement of vascular health, and the reduction of certain cardiovascular risk factors for hypertension.

In summary, the current study identified that upregulation of fibulin-3 and MMP expression was associated with hypertensive vascular remodeling in the thoracic aortas of SHRs. Fibulin-3 may function as a growth factor in the arteries, however increasing levels of fibulin-3 did not aggravate vascular remodeling of hypertensive aortas. In addition, fibulin-3 may reduce MMP-2, MMP-9 and oxidative stress in vascular remodeling in hypertension. Thus, it is suggested that increasing fibulin-3 may be beneficial to improve vascular health and reduce cardiovascular risk factors for hypertension.

\section{Acknowledgements}

The current study was supported in part by the Foundation of China Guangdong Science and Technology (grant nos. 2013B021800086 and 2013B021800128); the Foundation of Science and Technology of Bureau of Yuexiu District (Guangzhou, China) (grant no. 2014-WS-027); the National Natural Science Foundation of China (grant no. 81402465); and the Natural Science Foundation of Guangdong, China (grant no. 2015A030313583).

\section{References}

1. Argraves WS, Greene LM, Cooley MA and Gallagher WM Fibulins: Physiological and disease perspectives. EMBO Rep 4: $1127-1131,2003$.
2. Kobayashi N, Kostka G, Garbe JH, Keene DR, Bächinger HP, Hanisch FG, Markova D, Tsuda T, Timpl R, Chu ML and Sasaki T: A comparative analysis of the fibulin protein family. Biochemical characterization, binding interactions and tissue localization. J Biol Chem 282: 11805-11816, 2007.

3. Nagase H and Woessner JF Jr: Matrix metalloproteinases. J Biol Chem 274: 21491-21494, 1999.

4. Pérez-Rico C, Pascual G, Sotomayor S, Montes-Mollón MÁ, Trejo C, Sasaki T, Mecham R, Bellón JM and Buján J: Tropoelastin and fibulin overexpression in the subepithelial connective tissue of human pterygium. Am J Ophthalmol 151: 44-52, 2011.

5. Bergers G and Coussens LM: Extrinsic regulators of epithelial tumor progression: Metalloproteinases. Curr Opin Genet Dev 10: $120-127,2000$

6. Hu B, Thirtamara-Rajamani KK, Sim H and Viapiano MS: Fibulin-3 is uniquely upregulated in malignant gliomas and promotes tumor cell motility and invasion. Mol Cancer Res 7: 1756-1770, 2009.

7. Kim EJ, Lee SY, Woo MK, Choi SI, Kim TR, Kim MJ, Kim KC, Cho EW and Kim IG: Fibulin-3 promoter methylation alters the invasive behavior of non-small cell lung cancer cell lines via MMP-7 and MMP-2 regulation. Int J Oncol 40: 402-408, 2012.

8. Albig AR, Neil JR and Schiemann WP: Fibulins 3 and 5 antagonize tumor angiogenesis in vivo. Cancer Res 66: 2621-2629, 2006.

9. Lin ZW, Wang Z, Zhu GP, Li BW, Xie WL and Xiang DC: Hypertensive vascular remodeling was inhibited by Xuezhikang through the regulation of Fibulin-3 and MMPs in spontaneously hypertensive rats. Int J Clin Exp Med 8: 2118-2127, 2015.

10. Livak KJ and Schmittgen TD: Analysis of relative gene expression data using real-time quantitative PCR and the 2(-Delta Delta $\mathrm{C}(\mathrm{T})$ ) method. Methods 25: 402-408, 2001.

11. Muriel JM, Xu X, Kramer JM and Vogel BE: Selective assembly of fibulin-1 splice variants reveals distinct extracellular matrix networks and novel functions for perlecan/UNC-52 splice variants. Dev Dyn 235: 2632-2640, 2006.

12. Argraves WS, Tanaka A, Smith EP, Twal WO, Argraves KM, Fan D and Haudenschild CC: Fibulin-1 and fibrinogen in human atherosclerotic lesions. Histochem Cell Biol 132: 559-565, 2009.

13. Ström A, Olin AI, Aspberg A and Hultgårdh-Nilsson A: Fibulin-2 is present in murine vascular lesions and is important for smooth muscle cell migration. Cardiovasc Res 69: 755-763, 2006.

14. Hanada K, Vermeij M, Garinis GA, de Waard MC, Kunen MG, Myers L, Maas A, Duncker DJ, Meijers C, Dietz HC, et al: Perturbations of vascular homeostasis and aortic valve abnormalities in fibulin-4 deficient mice. Circ Res 100: 738-746, 2007.

15. Huang J, Davis EC, Chapman SL, Budatha M, Marmorstein LY, Word RA and Yanagisawa H: Fibulin-4 deficiency results in ascending aortic aneurysms: A potential link between abnormal smooth muscle cell phenotype and aneurysm progression. Circ Res 106: 583-592, 2010.

16. Spencer JA, Hacker SL, Davis EC, Mecham RP, Knutsen RH, Li DY, Gerard RD, Richardson JA, Olson EN and Yanagisawa H: Altered vascular remodeling in fibulin-5-deficient mice reveals a role of fibulin-5 in smooth muscle cell proliferation and migration. Proc Natl Acad Sci USA 102: 2946-2951, 2005.

17. Camaj P, Seeliger H, Ischenko I, Krebs S, Blum H, De Toni EN, Faktorova D, Jauch KW and Bruns CJ: EFEMP1 binds the EGF receptor and activates MAPK and Akt pathways in pancreatic carcinoma cells. Biol Chem 390: 1293-1302, 2009.

18. Feihl F, Liaudet L, Levy BI and Waeber B: Hypertension and microvascular remodelling. Cardiovasc Res 78: 274-285, 2008.

19. Longo GM, Xiong W, Greiner TC, Zhao Y, Fiotti N and Baxter BT: Matrix metalloproteinases 2 and 9 work in concert to produce aortic aneurysms. J Clin Invest 110: 625-632, 2002.

20. Yasmin SW, McEniery CM, Wallace S, Dakham Z, Pulsalkar P, Maki-Petaja K, Ashby MJ, Cockcroft JR and Wilkinson IB: Matrix metalloproteinase-9 (MMP-9), MMP-2, and serum elastase activity are associated with systolic hypertension and arterial stiffness. Arterioscler Thromb Vasc Biol 25: 372, 2005.

21. Klenotic PA, Munier FL, Marmorstein LY and Anand-Apte B: Tissue inhibitor of metalloproteinases-3 (TIMP-3) is a binding partner of epithelial growth factor-containing fibulin-like extracellular matrix protein 1 (EFEMP1). Implications for macular degenerations. J Biol Chem 279: 30469-30473, 2004.

22. Jahandideh F, Majumder K, Chakrabarti S, Morton JS, Panahi S, Kaufman S, Davidge ST and Wu J: Beneficial effects of simulated gastro-intestinal digests of fried egg and its fractions on blood pressure, plasma lipids and oxidative stress in spontaneously hypertensive rats. PLoS One 9: e115006, 2014. 
23. Schulz E, Gori T and Münzel T: Oxidative stress and endothelial dysfunction in hypertension. Hypertens Res 34: 665-673, 2011.

24. Tsutsui H, Kinugawa S and Matsushima S: Oxidative stress and heart failure. Am J Physiol Heart Circ Physiol 301: H2181-H2190, 2011

25. Bahrehmand F, Vaisi-Raygani A, Kiani A, Rahimi Z, Tavilani H, Ardalan M, Vaisi-Raygani H, Shakiba E and Pourmotabbed T: Matrix metalloproteinase 9 polymorphisms and systemic lupus erythematosus: Correlation with systemic inflammatory markers and oxidative stress. Lupus 24: 597-60, 2015.
26. Akhtar K, Broekelmann TJ, Miao M, Keeley FW, Starcher BC, Pierce RA, Mecham RP and Adair-Kirk TL: Oxidative and nitrosative modifications of tropoelastin prevent elastic fiber assembly in vitro. J Biol Chem 285: 37396-37404, 2010. 